\title{
Slip-Stick Mechanism in Training the Superconducting Magnets in the Large Hadron Collider
}

\author{
Pier Paolo Granieri, Clément Lorin, and Ezio Todesco
}

\begin{abstract}
Superconducting magnets can exhibit training quenches during successive powering to reaching nominal performance. The slip-stick motion of the conductors is considered to be one of the mechanisms of training. In this paper, we present a simple quantitative model where the training is described as a discrete dynamical system matching the equilibrium between the energy margin of the superconducting cable and the frictional energy released during the conductor motion. The model can be explicitly solved in the linearized case, showing that the short sample limit is reached via a power law. Training phenomena have a large random component. A large set of data of the large hadron collider magnet tests is postprocessed according to previously defined methods to extract an average training curve for dipoles and quadrupoles. These curves show the asymptotic power law predicted by the model. The curves are then fit through the model, which has two free parameters. The model shows good agreement over a large range, but it fails to describe the very initial part of the training.
\end{abstract}

Index Terms-Quench, slip-stick motion, superconducting accelerator magnets, training.

\section{INTRODUCTION}

$\mathbf{T}$ RAINING of superconducting magnets has been observed since the early stages of applied superconductivity [1], [2]. In many cases, magnets do not reach the nominal magnetic field performance at first powering, but only after successive powering. The magnet exhibits irrecoverable resistive transitions (quenches) at higher and higher currents, improving its performance until reaching a plateau, i.e., it "trains." The training speed can significantly vary, i.e., nominal performance can be reached with several quenches in some cases, or with only one or two quenches in others (see Fig. 1).

It is generally believed that training quenches are due to energy deposition in the coil due to mechanical reasons [2], i.e., cracks in the impregnation (when present) and/or frictional

Manuscript received June 6, 2011; revised July 8, 2011; accepted July 11, 2011. Date of publication September 1, 2011; date of current version September 28, 2011. This paper was recommended by Associate Editor L. Chiesa.

P. P. Granieri is with the Technology Department, European Organization for Nuclear Research, 1211 Geneva, Switzerland, and also with Ecole Polytechnique Federale of Lausanne, 1015 Lausanne, Switzerland (e-mail: Pier.Paolo.Granieri@cern.ch).

C. Lorin and E. Todesco are with the Technology Department, European Organization for Nuclear Research, 1211 Geneva, Switzerland (e-mail: Clement.Lorin@gmail.com; Ezio.Todesco@cern.ch).

Color versions of one or more of the figures in this paper are available online at http://ieeexplore.ieee.org.

Digital Object Identifier 10.1109/TASC.2011.2162727

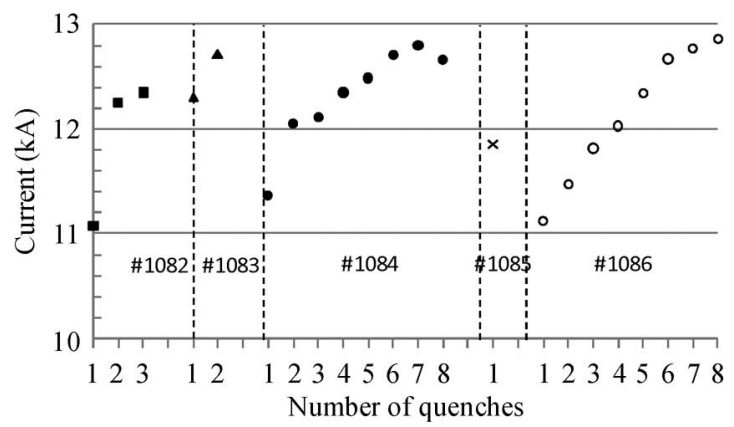

Fig. 1. Typical training curve of some LHC dipoles of the series production (nominal: $11.85 \mathrm{kA}$ ).

conductor motions on the order of micrometers can generate enough energy to drive the cable normal [3]-[5]. The motion is induced by the electromagnetic forces.

According to a phenomenological model based on frictional motion, one has slip-stick mechanism, i.e., during powering the coil's first slips against the support structure, releasing energy, and quenching the magnet, and then, it is kept (sticks) by friction in a new more stable position that can withstand higher electromagnetic forces. Hence, during successive powering, quench will occur at higher currents. The first aim of this paper is to translate this intuitive idea into a model to give a quantitative result about how the asymptotic performance is reached during training.

These kinds of phenomena have a large random component, i.e., training of a single magnet looks far from being a regular curve, particularly when it is fast (see Fig. 1). Some magnets can take a few quenches to reach nominal operating conditions, whereas others have no quench at all, both being built to the same design. A large statistical sample of identical magnets should allow deriving from experimental data the elements of an average behavior. This behavior is invisible on a single magnet, as it is swamped by the random part. Here, we single out an average training behavior from experimental data, where we take advantage of the large set relative to the large hadron collider (LHC) production, i.e., four homogeneous sets of about 400 magnets each, built by the same manufacturer [three lots of main dipoles (MB) and one of main quadrupoles (MQ)]. Postprocessing of the experimental data provides an average training for every lot and singles out regular patterns.

The second aim of this paper is the comparison between the training patterns found with the model and the average behavior extracted from the LHC data. 


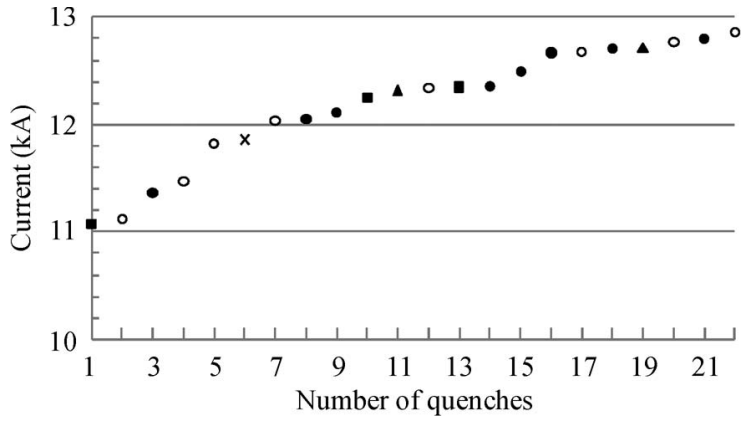

Fig. 2. Data of the previous figure reordered by the current, i.e., this plot represents the training of a string of magnets.

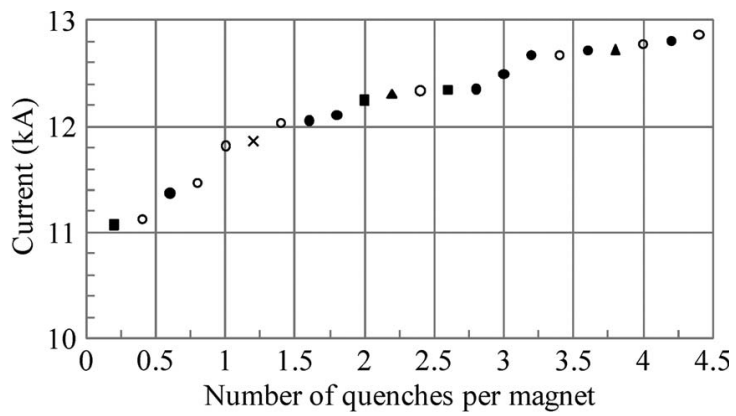

Fig. 3. Data of the previous figure divided by the number of magnets. Cumulative training curve of a string of magnets.

\section{Postprocessing of the Training Curves OF THE MAIN LHC MAGNETS}

Training data are usually plotted magnet by magnet (see Fig. 1). In our case, the large number of magnets allows presenting the training performance with cumulated plots, as proposed in [6]. In this plot, the whole set of same-design magnets is put together, reordering the quenches according to the current (see Fig. 2). The curve represents the training of the whole set of magnets as if they were powered in series, assuming no interaction between magnets and no detraining. The number of quenches is finally divided by the number of magnets; this curve gives a probability of quenching at a given current level (see Fig. 3).

The set of data we use consists of four lots of about 400 magnets each. The production of the 1232 LHC dipoles (plus 46 spares) was shared between three firms; the magnets were built according to the same design, with components provided by the European Organization for Nuclear Research (CERN), and tooling specific to each firm. The 392 quadrupoles, being less numerous than the dipoles, were produced by the same firm. Each magnet was tested at CERN at the operational temperature of $1.9 \mathrm{~K}$ and trained up to a current value ranging from 12 to $12.8 \mathrm{kA}$, corresponding to $85 \%-95 \%$ of the maximal performance.

Postprocessing takes into account that not all magnets were trained up to the same current during the tests [7], i.e., depending on the production rate, dipoles were required to reach 12 , 12.25 , or $12.85 \mathrm{kA}$ [8], and therefore, our sample contains less and less magnets when the current approaches the short sample limit [7].

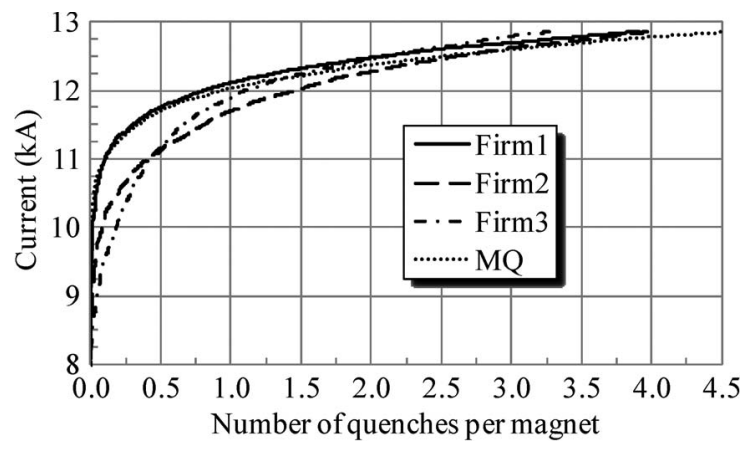

Fig. 4. Cumulated training curves for the LHC dipoles (subdivided by firms) and for the LHC quadrupoles.

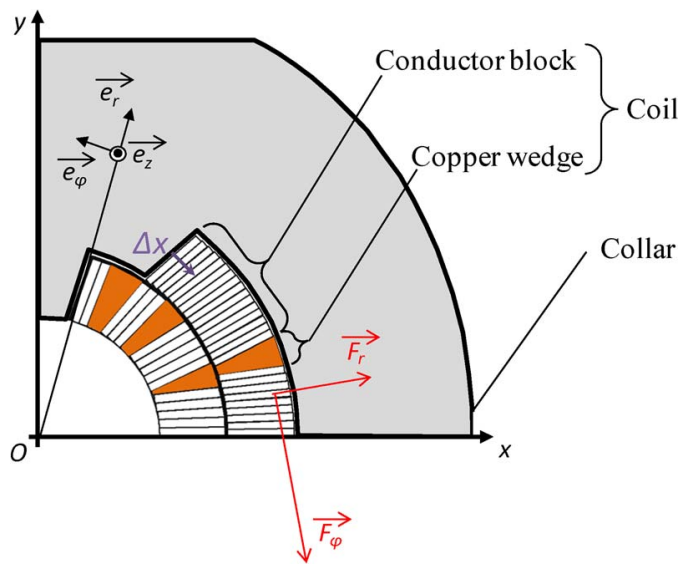

Fig. 5. Cross section of the LHC dipole (one quarter of aperture shown) and electromagnetic forces active during magnet powering.

In Fig. 4, we have shown the average training of a typical magnet of each firm extracted through the whole set of training data. It is interesting to note that in this plot, the number of quench takes noninteger values, i.e., it becomes a probability of quenching. For instance, the quadrupoles and Firm1 dipoles take 0.5 quenches to reach $11.8 \mathrm{kA}$; this means that having $N$ magnets, in average, they will reach $11.8 \mathrm{kA}$ with $N / 2$ quenches. Evidently, some of them will not quench, others will require one quench, and a few of them will require more than one, but the average is 0.5 quenches. This representation shows that the average training curve is extremely regular, as already observed in [6]. It is very tempting to fit this regular curve with a function [9], [10], but here, we aim at proposing a fit based on the physical description of the effect.

\section{Simplified Model of the Slip-Stick Motion}

\section{A. Frictional Energy}

During powering of the magnet, electromagnetic forces push on the coil (see Fig. 5). When the friction between the coil and the collar does not withstand the increasing forces, the coil quickly slips making sounds (creaks, cracks, and groans [11]); voltage spikes also occur. These spikes can be recorded [12]. Many observers have recorded the sounds and generally found a progressive increase in their amplitude and rate of incidence up to the point at which the magnet quenches [11]. We define $E_{f}$ as the energy released during a spike, i.e., a movement $\Delta x$ of 


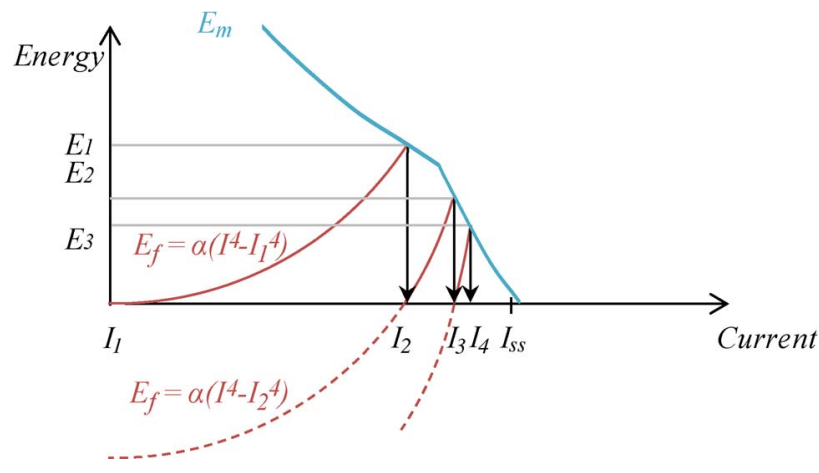

Fig. 6. Dynamic system based on a slip-stick motion giving a model for training in a superconducting magnet.

the coil. Following [13], we observe that the coil pushes toward the collars because of the radial component of the forces, whereas the displacement at the pole $\Delta x$ of the coil is related to the tangential component (see Fig. 5). Assuming a linear relation between force and displacement, $\Delta x$ is proportional to force, i.e., to the square of the current. Released energy $E_{f}$ is proportional to $\Delta x$ times the friction, and the friction is proportional to the radial force, i.e., to the square of the current. Therefore, $E_{f}$ is proportional to the fourth power of the current. The energy margin is the maximum energy that can be tolerated by the cable, still leading to a recovery. We assume that as long as the dissipated energy remains below the energy margin, there is no quench and that a quench occurs at $I_{1}$ as soon as the dissipated energy is larger than the margin. In the next powering, we assume that the frictional energy has been released up to the current $I_{1}$ of the previous quench, i.e.,

$$
\begin{aligned}
& E_{f 1}(I)=\alpha\left(I^{4}-I_{1}^{4}\right) \quad I>I_{1} \\
& E_{f 1}(I)=0 \quad I<I_{1}
\end{aligned}
$$

where $\alpha$ is a free parameter of the model.

This agrees with the well-known experimental observation that spike activities appear only beyond the maximum current reached in the magnet at the previous quench [14] (Kaiser effect [15], [16]).

When the energy margin is equal to the new frictional energy, we will have the second quench at $I_{2}$, and so on (see Fig. 6). This way, we have built a simple dynamical system that provides a model for the training. When powering from $I_{n}$ to $I_{n+1}$, the corresponding movement $\Delta x$ is split in several small movements that take place everywhere in the magnet during the ramp. Each movement is not able to quench the magnet since the energy is lower than the margin; this gives rise to the spikes and sounds. It is assumed that there is at least one position in the magnet where the coil is totally blocked over the whole range $\left[I_{n}, I_{n+1}\right]$ and where all the frictional energy is released at once at $I_{n+1}$, quenching the magnet.

\section{B. Energy Margin}

Energy margin $E_{m}$ is obtained by a numerical model taking into account the energy balance of the cable constituents [17], i.e., the superconducting strands, the helium filling the interstices among them, and the polyimide electrical insulation. The

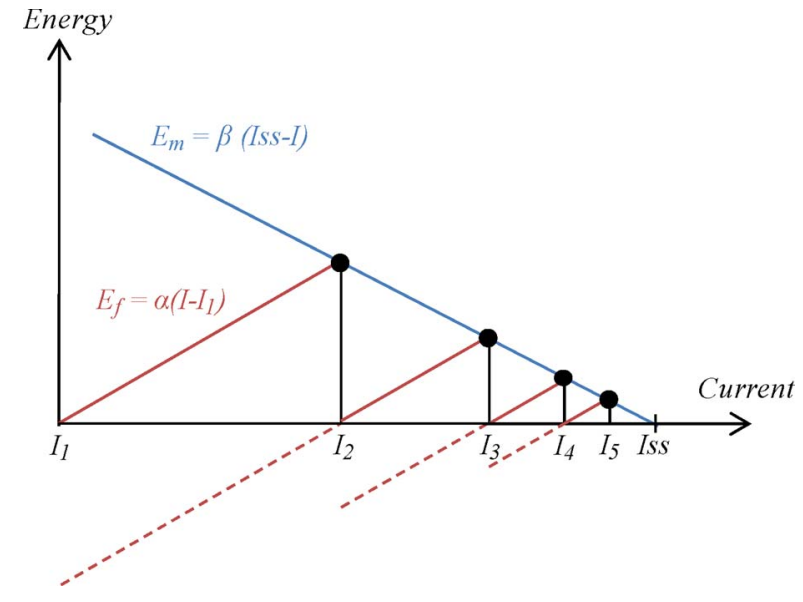

Fig. 7. Dynamical system based on a slip-stick motion giving a model for training in a superconducting magnet, i.e., linear case.

longitudinal cable dimension is neglected, but particular care is paid to the definition of the transient heat transfer between strands and helium, representing the mechanism that confers stability. This heat transfer model takes into account the different helium phases, i.e., He-II below the lambda temperature, He-I until saturation, nucleate boiling preceding the film boiling formation, and finally a completely gaseous phase. Transient perturbation is modeled by a square heating waveform, uniform over the cross section. The considered heating time is $0.5 \mathrm{~ms}$ [18]. Because of the fast heat deposition time, the mechanism of the He-II heat transfer between the cable and the surrounding helium bath through the insulation channels does not have a significant impact on the cable stability and can therefore be neglected. The calculations refer to the most critical cable, i.e., the one located in the higher field region.

The energy margin curve obtained with the described model is reported in Fig. 6. It is worth noting that the helium contribution to stability is larger at low currents, where smaller Joule heating and the larger temperature margin lead to a long time (on the order of $0.1 \mathrm{~s}$ ) the system takes to decide whether to quench or not. Meanwhile, helium can behave as a good thermal sink, increasing its temperature and going through all the mentioned phases. For high-current levels, the decision time is on the order of the heating time. The helium temperature before the thermal runaway starts is below saturation; therefore, it does not even enter the boiling phases and its contribution to stability is rather limited. At the highest currents, helium does not even reach the lambda transition. Hence, the cable cannot take advantage of the corresponding peak of specific heat; this justifies the change of slope that can be observed in Fig. 6.

\section{Linear Model}

When the current gets closer to the short sample limit, the energy margin is a linear decreasing function of $I$ that vanishes at the short sample current $I=I_{s s}$ (see Fig. 7), which is defined as

$$
E_{m}(I)=\beta\left(I_{s s}-I\right)
$$




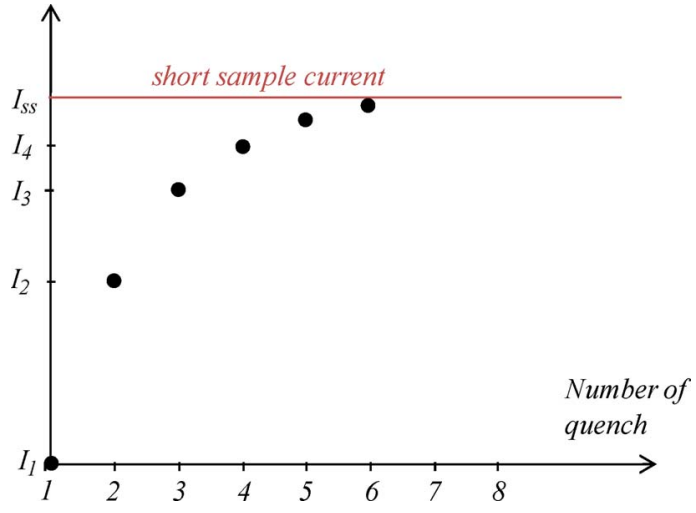

Fig. 8. Training in the case of speed $\alpha / \beta=1$, i.e., each quench half of the gap to the short sample is gained.

where $\beta$ is a free parameter. Moreover, the frictional energy becomes linear because $\Delta I$ becomes small, i.e.,

$$
E_{f}(I)=\alpha\left(I-I_{n}\right)
$$

and the whole model can be explicitly solved as

$$
I_{n+1}=I_{s s}-\left(I_{s s}-I_{1}\right) \exp \left(-n / n_{a}\right)
$$

where

$$
n_{a}=\frac{1}{\ln (1+\beta / \alpha)} .
$$

On the other hand, equivalently

$$
I_{s s}-I_{n+1}=\left(I_{s s}-I_{1}\right)\left(1+\frac{\beta}{\alpha}\right)^{-n} .
$$

The aforementioned expression simply means that the difference between the quench current and the short sample current tends to zero with a power law. The ratio $\alpha / \beta$ gives the speed of training. For instance, if $\alpha / \beta=1$, one gains half of the gap to the short sample current at each quench, i.e., if the first quench is at $80 \%$ of the short sample, one will have $I_{2} / I_{s s}=0.9$, $I_{3} / I_{s s}=0.95, I_{4} / I_{s s}=0.975$, and so on (see Fig. 8).

If $\alpha / \beta=3$, the speed is slower, and one gains one quarter of the gap to the short sample at each quench, i.e., $I_{2} / I_{s s}=0.85$, $I_{3} / I_{s s}=0.8875$, and so on. In general, we gain $1 /(1+\alpha / \beta)$ of the gap to the short sample current after every quench. This behavior implies that in a semilog scale, the training curves are linear with respect to the quench number $n$, i.e.,

$$
\ln \left(\frac{I_{s s}-I_{n+1}}{I_{s s}}\right)=\ln \left(\frac{I_{s s}-I_{1}}{I_{s s}}\right)-n \ln \left(1+\frac{\beta}{\alpha}\right) .
$$

\section{Model Versus Measurements}

The linear approximation above provides a very simple tool to check our model versus experimental data; for a large number of quenches, training curves should be linear on this scale. The plot for one set of the LHC data is shown in Fig. 9, showing that the asymptotic linear behavior is confirmed. The initial part of the training has a larger curvature, i.e., the training starts at lower current values and trains faster than the linear model. This is particularly evident for rare quenches in the initial part (quench probability smaller than 0.5 , i.e., the first

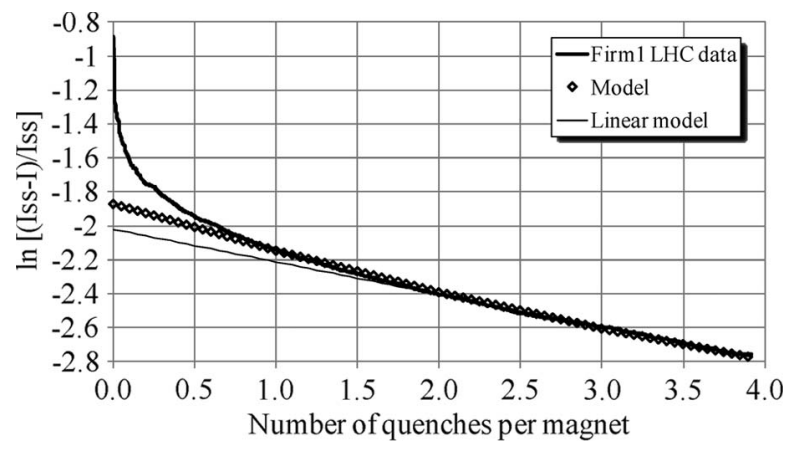

Fig. 9. Cumulative curve of training for the LHC dipoles, Firm1: measurements versus model in semi log scale.

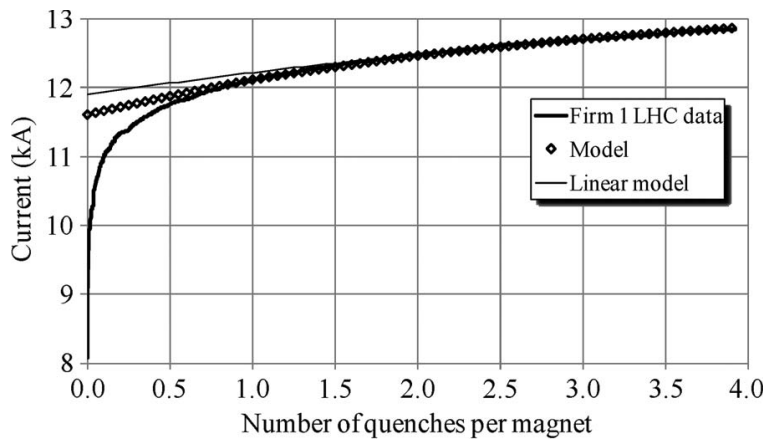

Fig. 10. Cumulative curve of training for the LHC dipoles, Firm1: measurements versus model.

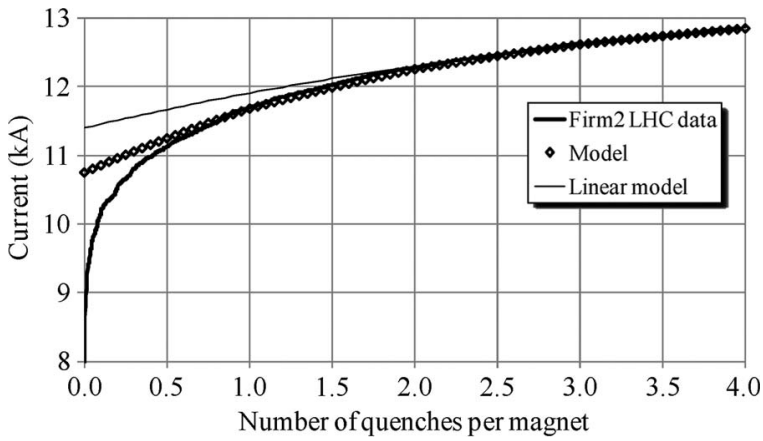

Fig. 11. Cumulative curve of training for the LHC dipoles, Firm2: measurements versus model.

quench of the worst half of the magnets). Using the complete model for the energy margin and adding the nonlinearity to the frictional energy, according to (1), we push the training curve in the right direction with respect to the experimental data; the early training is better fitted but not enough (see Figs. 9-13). For instance, in Fig. 10, the fit begins around 0.8 quenches per magnet instead of 1.6 quenches per magnet for the linear model. Therefore, the model accounts for a significant fraction of the training curve, but it fails in the initial part. One has to also point out that the model assumes that quenches are located in the higher field region; this is not always the case, since quenches can be distributed in different parts of the coil.

The model has two free parameters, i.e., the initial quench current $I_{1}$ and the speed $\alpha / \beta$. While it does not seem to be justified from the physical point of view, it is tempting to consider modifying the model by freeing the exponent of the frictional energy. With this approach, one can indeed obtain a better fit, as shown in the Appendix. 


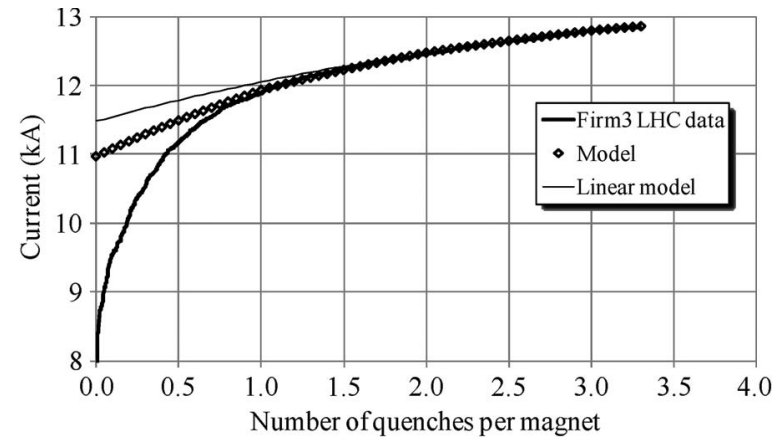

Fig. 12. Cumulative curve of training for the LHC dipoles, Firm3: measurements versus model.

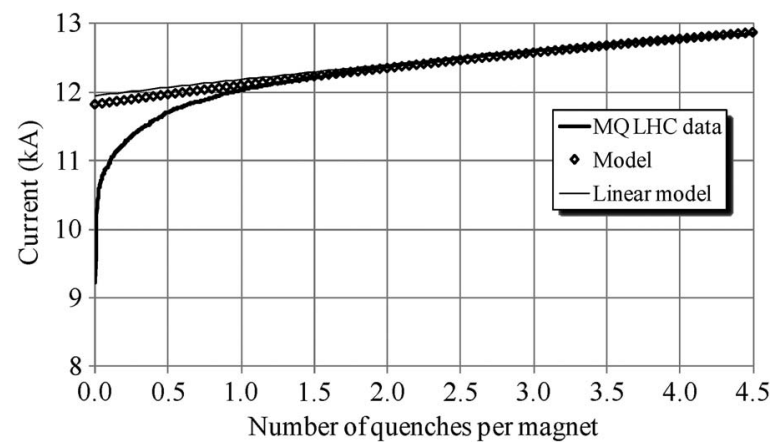

Fig. 13. Cumulative curve of training for the LHC quadrupoles, MQ: measurements versus model.

\section{CONCLUSION}

The large amount of magnets built for the LHC provides a unique opportunity to study training in superconducting magnets. In this paper, we have used a method to build cumulative training curves of a set of homogeneous magnets, extracting an average behavior. We then constructed a hypersimplified model based on the equilibrium between the energy margin and the energy dissipated by friction taking as a hypothesis slip-stick motion. An interesting feature of this model is that it gives an explicit expression for the training curves in their asymptotic regime, i.e., for a large number of quenches; the short sample current is approached via a power law in the number of quenches [see (6)]. The model has two free parameters, i.e., the initial quench and the speed of training, which is related to the frictional energy and to the energy margin. Experimental data of four different sets of about $400 \mathrm{LHC}$ magnets each (three sets of dipoles and one set of quadrupoles) confirm this power law. The initial part of the training does not fit with the model, the training being faster than expected.

\section{APPENDIX}

Here, we consider a model with a frictional energy having a free exponent $p$, i.e.,

$$
E_{f 1}(I)=\alpha\left(I^{p}-I_{1}^{p}\right) .
$$

The comparison to experimental data shows remarkable agreement with exponents ranging from 10 to 20 (see Figs. 14-17). Since we do not see any physical justification

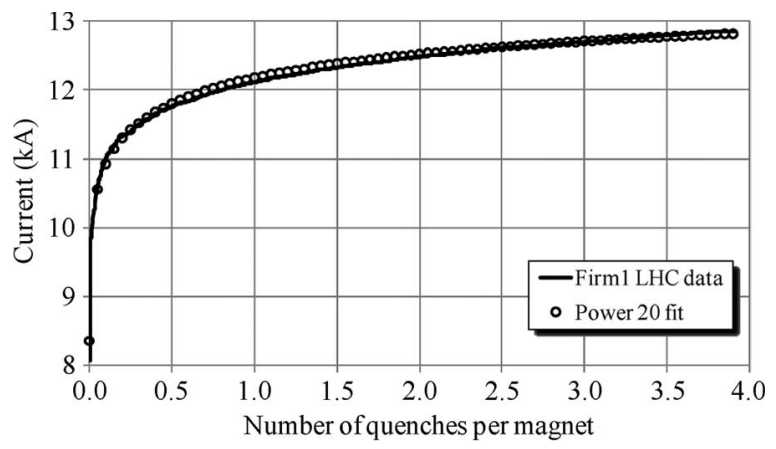

Fig. 14. Cumulative curve of training for the LHC dipoles, Firm1, versus generalized model with exponent 20 .

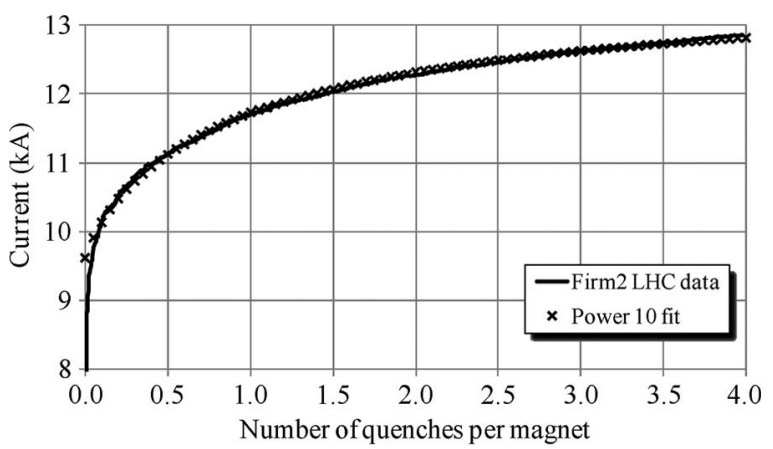

Fig. 15. Cumulative curve of training for the LHC dipoles, Firm1, versus generalized model with exponent 10 .

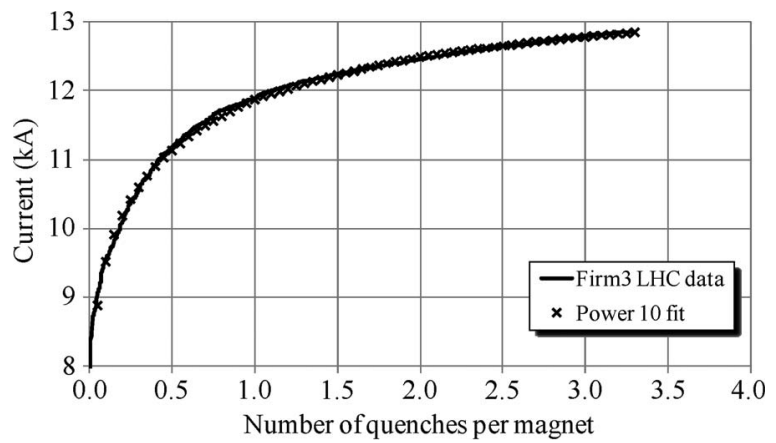

Fig. 16. Cumulative curve of training for the LHC dipoles, Firm3, versus generalized model with exponent 10 .

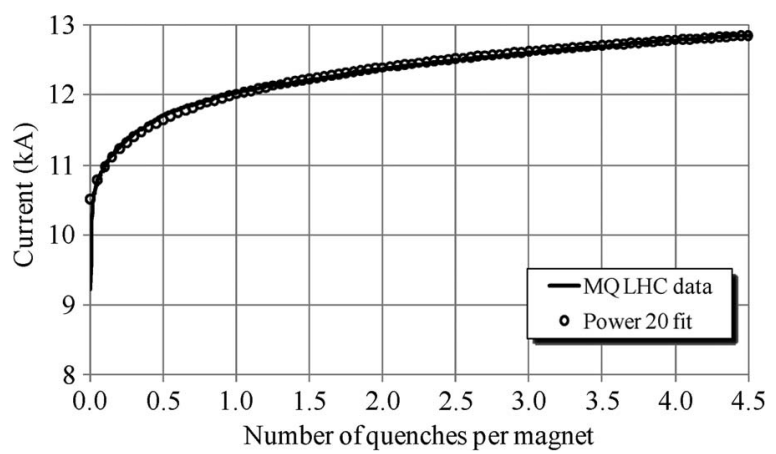

Fig. 17. Cumulative curve of training for the LHC quadrupoles, MQ, versus generalized model with exponent 20.

for these large exponents, we consider this feature more of a curiosity than a fact. Anyway, it is rather striking to see a threeparameter curve fitting the experimental data so precisely. 


\section{ACKNOWLEDGMENT}

The authors would like to thank M. Pojer and $\mathrm{V}$. Remondino for providing the data of the dipoles and quadrupoles, respectively, A. Siemko and all colleagues involved in magnet testing, and L. Bottura and T. Taylor for reading the manuscript and useful comments. The authors would like to give special thanks to A. Dallocchio for triggering an interesting discussion on training, which led to this study.

\section{REFERENCES}

[1] P. F. Chester, W. T. Norris, and Z. Croitoru, "Superconducting magnets for M.H.D. generators [and discussion]," Philos. Trans. Roy. Soc. London A, Math. Phys. Sci., vol. 261, no. 1123, pp. 558-579, Jul. 1967.

[2] A. V. Tollestrup, "Care and training of superconducting magnets," IEEE Trans. Magn., vol. MAG-17, no. 1, pp. 863-872, Jan. 1981.

[3] E. Acerbi, F. Alessandria, and L. Rossi, "Possible movements of the Milan superconducting coils under the influence of mechanical stresses," in Proc. 10th Int. Conf. Cyclotrons Appl., East Lansing, MI, Apr. 29-May 3 1984, pp. 71-74.

[4] S. Caspi, W. Gilbert, W. Hassenzahl, G. Lamberston, R. Meuser, J. Rechen, R. Schafer, and R. Warren, "Mechanical and helium cooling factors in dipole magnet training," IEEE Trans. Magn., vol. MAG-17, no. 1, pp. 443-446, Jan. 1981.

[5] W. S. Gilbert and W. V. Hassenzahl, "1.8 K conditioning (non-quench training) of a model of SSC dipoles," IEEE Trans. Magn., vol. MAG-23, no. 2, pp. 1229-1232, Mar. 1987.

[6] B. Bellesia, N. Catalan Lasheras, and E. Todesco, "Magnet (re)training," in Proc. Chamonix Workshop, 2009, pp. 264-270.

[7] C. Lorin and E. Todesco, "Training of the main LHC dipoles. Episode I: Evolution during production, quench location, and storage time," CERN, Geneva, Switzerland, Tech. Rep. CERN-ATS-2010-164, 2010.

[8] P. Pugnat and A. Siemko, "Review of quench performance of LHC main superconducting magnets," IEEE Trans. Appl. Supercond., vol. 17, no. 2, pp. 1091-1096, Jun. 2007.

[9] C. Lorin, A. Siemko, E. Todesco, and A. Verweij, "Predicting the quench behavior of the LHC dipoles during commissioning," IEEE Trans. Appl. Supercond., vol. 20, no. 3, pp. 135-139, Jun. 2010.

[10] A. Verweij, "Training the dipoles," in Proc. Chamonix Workshop, 2009, pp. $25-28$

[11] M. N. Wilson, Superconducting Magnets. London, U.K.: Oxford Univ. Press, 1983, p. 85.

[12] D. Leroy, J. Krzywinski, V. Remondino, L. Walckiers, and R. Wolf, "Quench observation in LHC superconducting one meter long dipole models by field perturbation measurements," IEEE Trans. Appl. Supercond., vol. 3, no. 1, pp. 781-784, Mar. 1993.

[13] V. E. Keilin, "Explanation of main features of superconducting windings training by balance of acting and permissible disturbances," IEEE Trans. Appl. Supercond., vol. 3, no. 1, pp. 297-300, Mar. 1993.

[14] G. Pasztor and C. Schmidt, "Dynamic stress effects in technical superconductors and the "training" problem of superconducting magnets," J. Appl. Phys., vol. 49, no. 2, pp. 886-899, Feb. 1978.
[15] J. Kaiser, "Erkenntnisse und Folgerungen aus der Messung von Geräuschen bei Zugbeanspruchung von metallischen Werkstoffen," Archiv für das Eisenhüttenwesen, vol. 24, 1953.

[16] T. J. Fowler, "Acoustic emission of fiber reinforced plastics," J. Tech. Council Amer. Soc. Civil Eng., vol. 105, no. 2, pp. 281-289, Dec. 1979.

[17] P. P. Granieri, M. Calvi, P. Xydi, B. Baudouy, D. Bocian, L. Bottura, M. Breschi, and A. Siemko, "Stability analysis of the LHC cables for transient heat depositions," IEEE Trans. Appl. Supercond., vol. 18, no. 2, pp. 1257-1262, Jun. 2008

[18] P. Pugnat, B. Khomenko, A. Rijllart, S. Sanfilippo, and A. Siemko, "Statistical diagnosis method of conductor motions in superconducting magnets to predict their quench performance," IEEE Trans. Appl. Supercond., vol. 11, no. 1, pp. 1705-1708, Mar. 2001.

Pier Paolo Granieri received the M.S. degree in mechanical engineering from the University of Bologna, Bologna, Italy, in 2008. He is currently working toward the Ph.D. degree in applied physics in the Particle Accelerator Physics Laboratory (LPAP), Swiss Federal Institute of Technology (EPFL), Lausanne, Switzerland.

Since 2006, he has been with the European Organization for Nuclear Research (CERN), Geneva, Switzerland. He has been working for four years in the Magnet Group on the stability of superconducting cables and thermal characterization of helium-permeable cable insulation. Since 2011, he has been a Senior Research Fellow in the Cryogenics Group, where his main research interest is the heat transfer in superconducting magnets.

Clément Lorin received the Master's degree from the Ecole Nationale Supérieure d'Electricité et de Mécanique, Vandoeuvre-lès-Nancy, France, in 2005 and the Ph.D. degree from the Institut National Polytechnique de Lorraine, Vandoeuvre-lès-Nancy, in 2008, both in electrical engineering.

During his thesis, he has worked on superconducting devices for magnetic compensation of gravity, with applications to the next generation of cryogenic engines for rockets. From 2009 to 2012, he was a Postdoctoral Research Fellow with the European Organization for Nuclear Research (CERN), Geneva, Switzerland, where he worked on superconducting magnets.

Ezio Todesco received the Master and Ph.D. degrees from the University of Bologna, Bologna, Italy, in 1989 and 1994, respectively, both in physics.

He has been working on nonlinear dynamics of conservative systems, focusing on applications of perturbative theory to particle motion in accelerators, beam stability, and resonances. Since 1998, he has been with the European Organization for Nuclear Research, Geneva, Switzerland, where he has been working in modeling and analyzing the field quality of the main magnets of the Large Hadron Collider (LHC). Since 2009, he has been in charge of the magnetic model of the LHC and its implementation for beam commissioning. Currently, he is working on magnet design for the LHC luminosity upgrade. 